\title{
CONCEPT DESIGN OF A MULTIWAVELENGTH AEROSOL LIDAR SYSTEM WITH MITIGATED DIATTENUATION EFFECTS AND DEPOLARIZATION-MEASUREMENT CAPABILITY
}

\author{
Adolfo Comerón ${ }^{1 *}$, Michaël Sicard ${ }^{1,2}$, Eric Vidal ${ }^{1}$, Rubén Barragán ${ }^{1}$, Constantino Muñoz ${ }^{1}$, \\ Alejandro Rodríguez ${ }^{1}$, Jordi Tiana-Alsina ${ }^{1}$, Francesc Rocadenbosch ${ }^{1,2}$, David García-Vizcaíno ${ }^{1}$ \\ ${ }^{1}$ RSLab, Department of Signal Theory and Communications, Universitat Politècnica de Catalunya, 08034 \\ Barcelona, Spain, *Email:comeron@tsc.upc.edu \\ ${ }^{2}$ Institut d'Estudis Espacials de Catalunya (IEEC), Barcelona, Spain
}

\begin{abstract}
It is known that the retrieval of aerosol extinction and backscatter coefficients from lidar data acquired through so-called total-power channels intended to measure the backscattered power irrespective of the polarization - can be adversely affected by varying depolarization effects produced by the aerosol under measurement. This effect can be particularly noticeable in advanced multiwavelength systems, where different wavelengths are separated using a system of dichroic beam splitters, because in general the reflection and transmission coefficients of the beam splitters will be different for fields with polarization parallel or perpendicular to the incidence plane. Here we propose a setup for multiwavelength aerosol lidars alleviating diattenuation effects due to changing depolarization conditions while allowing measure linear depolarization.
\end{abstract}

\section{INTRODUCTION}

Advanced aerosol-lidar systems use several transmitted wavelengths and several receiving channels, both elastic and Raman, for the rangeresolved measurement of the extinction and backscatter coefficient of atmospheric aerosols at different wavelengths. From the behavior of these optical coefficients as a function of the wavelength, microphysical properties of the particles can be inferred [1]. The addition of depolarization channels allows further microphysical characterization of the aerosols by providing information about the shape of the particles contributing to the backscatter [2]-[4]. In such advanced systems some of the channels are total-

\footnotetext{
${ }^{1}$ For the sake of simplicity in the terminology, we will talk henceforth only about transmittance, since reflectance plays exactly the same role in the equations
}

power channels, intended to receive the total power scattered from the atmosphere at a given wavelength; in some configurations, total-power channels can also be part of the arrangement for measuring depolarization [5]. To separate the different wavelengths in the lidar receiver setup, dichroic beam splitters are usually employed to direct the different incoming wavelengths to the corresponding receivers. Nevertheless the reflectance and transmittance ${ }^{1}$ of beam splitters depend in general on the polarization of the incident radiation. Because of this, for the same amount of collected backscattered power, the power reaching the photodetector of a total power channel can vary noticeably depending on the depolarization produced by the aerosol contributing to the backscatter. The response of the beam splitters to an arbitrarily polarized wave can be expressed in terms of the responses to waves with linear polarizations respectively parallel and perpendicular to the plane of incidence (henceforth called $\mathrm{p}$ - and s-polarized wave respectively) [6],[7]. It has been shown [7] that, for the usual case of lidars transmitting linearly-polarized beams, when the transmission ratio (the ratio of the receiver transmittance for the polarization to the transmittance for the s polarization) exceeds the interval $(0.85,1.15)$ the retrieval of the aerosol backscatter coefficient in the presence of mineral dust can be biased by more than $5 \%$.

Making the transmission ratio close to 1 to reduce the effect of varying polarization may have a big impact on the cost of the lidar receiver system, as it requires custom-coated beam splitters [4]. Nevertheless, if i) the different wavelengths transmitted all have linear polarizations parallel or

that give the power reaching a photodetector and has the same formal dependence on the polarization of the incident wave as the transmittance. 
perpendicular between themselves, ii) the backscattering medium does not change the polarized part of the returned radiation with respect to the polarization of the transmitted beams [8] [10], nor does it the part of the optical receiving system before the beam splitters, and iii) all the beam splitters in the receiver setup are arranged in such a way that the incidence plane is the same for all of them, the sensitivity of the receiver to the depolarization produced by the medium can be suppressed by orientating the incidence plane to be at a $45^{\circ}$ angle with respect the polarization direction of the polarized part of the return radiation, irrespective of the transmission ratio. In this case the depolarization information after transmission or reflections is found in the $3^{\text {rd }}$ and $4^{\text {th }}$ components of the Stokes vector, whence it can be retrieved with a quarter-wave plate and linear polarizer arrangement.

\section{PROPOSED SETUP}

It can be easily shown that, if they share the same incidence plane, the Mueller matrix of a system of $\mathrm{N}$ cascaded beam splitters where the $\mathrm{i}$-th element has a (complex) field transmittance $t_{p i}$ in the $\mathrm{p}$ plane and $t_{s i}$ in the s-plane is

$[M]=\frac{1}{2}\left[\begin{array}{cccc}\left|t_{p}\right|^{2}+\left|t_{s}\right|^{2} & \left|t_{p}\right|^{2}-\left|t_{s}\right|^{2} & 0 & 0 \\ \left|t_{p}\right|^{2}-\left|t_{s}\right|^{2} & \left|t_{p}\right|^{2}+\left|t_{s}\right|^{2} & 0 & 0 \\ 0 & 0 & 2 \operatorname{Re}\left[t_{p}^{*} t_{s}\right] & 2 \operatorname{Im}\left[t_{p}^{*} t_{s}\right] \\ 0 & 0 & -2 \operatorname{Im}\left[t_{p}^{*} t_{s}\right] & 2 \operatorname{Re}\left[t_{p}^{*} t_{s}\right]\end{array}\right]$,

where $t_{p}=\prod_{i=1}^{N} t_{p i}$ and $t_{s}=\prod_{i=1}^{N} t_{s i}$.

If we assume that the light entering the optical setup is a combination of linearly polarized light and completely depolarized light, as would be the case if the polarization of the transmitted light is linear and the depolarizing scatterers do not have a preferred orientation [8]-[10], the Stokes vector describing the intensity and polarization state for that radiation will be [11]

$$
\left[s_{i}\right]^{T}=\left[\begin{array}{llll}
I & I_{\|} \cos 2 \gamma & I_{\|} \sin 2 \gamma & 0
\end{array}\right],
$$

where $I$ is the total intensity of the light, $I_{\|}$is the intensity of the linearly polarized part, and $\gamma$ is the angle of the linear polarization direction with respect to a reference direction perpendicular to the propagation one. If the reference direction is taken parallel to the incidence plane of the beam splitters, the Stokes vector at the setup output is found as $\left[s_{o}\right]=[M]\left[s_{i}\right]$,

$$
\left[s_{o}\right]=\frac{1}{2}\left[\begin{array}{c}
\left(\left|t_{p}\right|^{2}+\left|t_{s}\right|^{2}\right) I+\left(\left|t_{p}\right|^{2}-\left|t_{s}\right|^{2}\right) I_{\|} \cos 2 \gamma \\
\left(\left|t_{p}\right|^{2}-\left|t_{s}\right|^{2}\right) I+\left(\left|t_{p}\right|^{2}+\left|t_{s}\right|^{2}\right) I_{\|} \cos 2 \gamma \\
2 \operatorname{Re}\left[t_{p}^{*} t_{s}\right] I_{\|} \sin 2 \gamma \\
-2 \operatorname{Im}\left[t_{p}^{*} t_{s}\right] I_{\|} \sin 2 \gamma
\end{array}\right],
$$

which for $\gamma=45^{\circ}$ or $\gamma=135^{\circ}$ reduces to

$$
\left[s_{o}\right]=\frac{1}{2}\left[\begin{array}{c}
\left(\left|t_{p}\right|^{2}+\left|t_{s}\right|^{2}\right) I \\
\left(\left|t_{p}\right|^{2}-\left|t_{s}\right|^{2}\right) I \\
2 \operatorname{Re}\left[t_{p}^{*} t_{s}\right] I_{\|} \\
-2 \operatorname{Im}\left[t_{p}^{*} t_{s}\right] I_{\|}
\end{array}\right]
$$

Eq. (1) shows that the transmitted power $\left(1^{\text {st }}\right.$ element of the Stokes vector) has become insensitive to the depolarization ratio. The depolarization information is found in the $3^{\text {rd }}$ and $4^{\text {th }}$ components of the Stokes vector (the power of

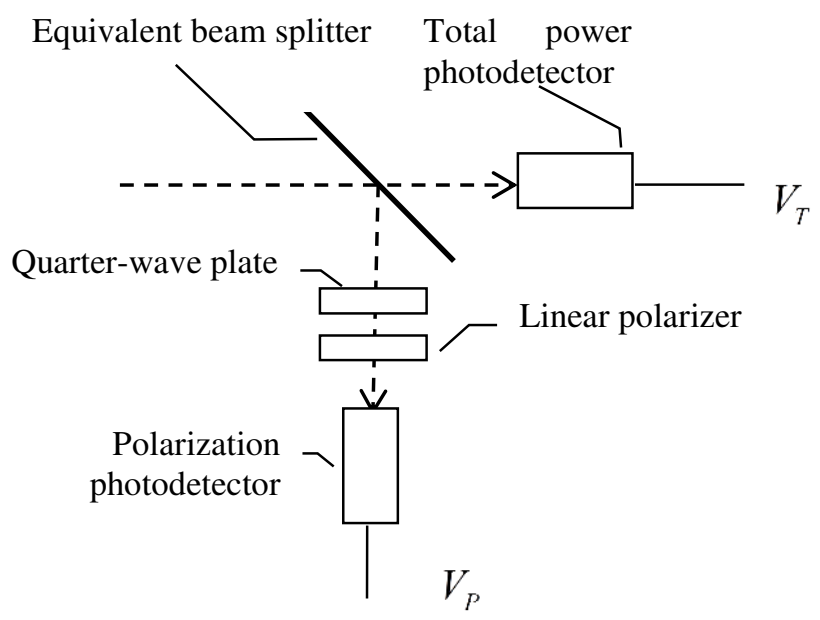

Fig. 1. Setup for measuring the calibrated volume depolarization ratio.

the depolarized light is $\left.I_{d}=I-I_{\|}\right)$, whence it can be extracted and calibrated using the setup outlined in fig. 1, where the equivalent beam splitter represents any combination of beam splitters with 
the sole condition that they share their incidence planes.

\section{DEPOLARIZATION CALIBRATION}

Calibration of the depolarization ratio is crucial for quantitative measurements. Assuming the (linearly) polarized part of the incident light has the polarization direction at $45^{\circ}$ with respect to the incidence plane, the Stokes vector of the transmitted beam will have the form given by Eq. (1). As the first component of the Stokes vector corresponds to the total power of the beam and it is proportional to the total incoming power $I$, we will designate the detector measuring the power of the transmitted beam as the total-power photodector. At its output we will have a voltage

$$
V_{T}=V_{T}^{*} \frac{1}{2}\left(\left|t_{p}\right|^{2}+\left|t_{s}\right|^{2}\right) I,
$$

with $V_{T}^{*}$ the voltage responsivity of the photodetector. The Stokes vector of the reflected beam, before passing through the quarter-wave plate and the linear polarizer, will have the form given by Eq. (1), with the reflection coefficients $r_{p}, r_{s}$ substituted for the respective transmission ones.

The Mueller matrix of the cascaded quarter-wave plate and linear polarizer can be shown to be $\left[M_{e \alpha \theta}\right]=$

$$
\frac{1}{2}\left[\begin{array}{cc}
1 & \cos 2 \alpha \cos 2(\alpha-\theta) \\
\cos 2 \theta & \cos 2 \alpha \cos 2 \theta \cos 2(\alpha-\theta) \\
\sin 2 \theta & \cos 2 \alpha \sin 2 \theta \cos 2(\alpha-\theta) \\
0 & 0
\end{array}\right.
$$

$$
\begin{aligned}
& \sin 2 \alpha \cos 2(\alpha-\theta) \\
& \sin 2 \alpha \cos 2 \theta \cos 2(\alpha-\theta) \quad \cos 2 \theta \sin 2(\alpha-\theta) \\
& \sin 2 \alpha \sin 2 \theta \cos 2(\alpha-\theta) \quad \sin 2 \theta \sin 2(\alpha-\theta) \\
& \frac{0}{R_{4545}=\frac{1}{2}\left(R_{00}+R_{090}\right)+\sqrt{R_{00} R_{090}} \cos \left(\varphi_{s}-\varphi_{p}\right) \frac{I_{I I}}{I},(5)}
\end{aligned}
$$

with $\alpha$ the angle between the quarter-wave plate fast axis and the p-plane and $\theta$ the angle between the polarizer transmission axis and the p-plane.

By setting $\alpha=0, \theta=0$, the Stokes vector of the light exiting the ensemble is

$$
\left[s_{r 00}\right]^{T}=\frac{1}{2}\left[\left|r_{p}\right|^{2} I \quad\left|r_{p}\right|^{2} I \quad 0 \quad 0\right],
$$

and the polarization photodetector will yield an output voltage

$$
V_{p 00}=V_{p}^{*} \frac{1}{2}\left|r_{p}\right|^{2} I
$$

with $V_{p}^{*}$ the voltage responsivity of the polarization photodetector. By making the ratio between $V_{p 00}$ and $V_{T}$ we obtain the coefficient

$$
R_{00}=\frac{V_{p}^{*}}{V_{T}^{*}} \frac{\left|r_{p}\right|^{2}}{\left|t_{p}\right|^{2}+\left|t_{s}\right|^{2}} .
$$

Likewise, by setting $\alpha=0, \theta=90^{\circ}$, we will obtain an output voltage of the polarization photodetector that divided by $V_{T}$ will result in the coefficient

$$
R_{090}=\frac{V_{p}^{*}}{V_{T}^{*}} \frac{\left|r_{s}\right|^{2}}{\left|t_{p}\right|^{2}+\left|t_{s}\right|^{2}} .
$$

Dividing the output of the polarization photodetector when $\alpha=45^{\circ}, \theta=45^{\circ}$ by $V_{T}$ we obtain a coefficient

$$
\begin{aligned}
& R_{4545}= \\
& \frac{1}{2} \frac{V_{p}^{*}\left[\left(\left|r_{p}\right|^{2}+\left|r_{s}\right|^{2}\right)+2\left|r_{p}\right|\left|r_{s}\right| \cos \left(\varphi_{s}-\varphi_{p}\right) \frac{I_{I}}{I}\right]}{V_{T}^{*}\left(\left|t_{p}\right|^{2}+\left|t_{s}\right|^{2}\right)},
\end{aligned}
$$

which using Eqs. (3)and (4) can be written as

with $\varphi_{s}$ and $\varphi_{p}$ the phases of the complex reflection coefficients $r_{s}$ and $r_{p}$ respectively.

Likewise setting $\alpha=45^{\circ}, \theta=0$ and dividing the output of the polarization photodetector by $V_{T}$ we obtain the coefficient

$$
R_{450}=\frac{1}{2}\left(R_{00}+R_{090}\right)-\sqrt{R_{00} R_{090}} \sin \left(\varphi_{s}-\varphi_{p}\right) \frac{I_{\|}}{I} .
$$

The diphase $\varphi_{s}-\varphi_{p}$ can be calculated from Eqs. (5) and (6): 


$$
\tan \left(\varphi_{s}-\varphi_{p}\right)=\frac{R_{00}+R_{090}-2 R_{450}}{2 R_{4545}-R_{00}-R_{090}},
$$

and the quadrant uncertainty can be removed by checking the signs of the numerator and the denominator. Note that, to find $\varphi_{s}-\varphi_{p}$ from Eq. (7), the ratio $I_{\|} / I$ must be the same in Eqs. (5) and (6); therefore the measurements of $R_{4545}$ and of $R_{450}$ must be very close in time, or else they must be made on an aerosol-free region, where the depolarization ratio does not change.

Once the diphase has been found, the ratio $I_{\|} / I$ can be calculated from

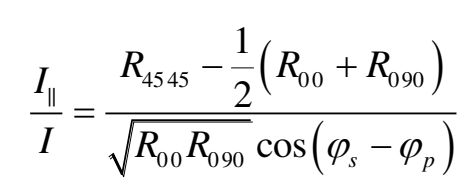

or from

$$
\frac{I_{\|}}{I}=\frac{\frac{1}{2}\left(R_{00}+R_{090}\right)-R_{450}}{\sqrt{R_{00} R_{090}} \sin \left(\varphi_{s}-\varphi_{p}\right)},
$$

depending respectively on whether $\cos \left(\varphi_{s}-\varphi_{p}\right)$ or $\sin \left(\varphi_{s}-\varphi_{p}\right)$ has the maximum absolute value.

\section{SUMMARY AND OUTLOOK}

A setup has been proposed that allows alleviating the diattenuation-induced uncertainties while maintaining the depolarization measurement capability in multiwavelength aerosol lidar systems. Underlying assumptions are that all the transmitted wavelengths are linearly polarized with polarizations parallel or perpendicular to each other and that the aerosol particles are randomly oriented. Further work is necessary to study the sensitivity of the system to non-idealities such as angle tolerances and imperfect linear polarization in the transmitted beams.

\section{ACKNOWLEDGEMENTS}

This work has been supported by the following projects and grants: European Union $7^{\text {th }}$ Framework Programme grant agreement No. 262254 (ACTRIS), Spanish Ministry for Economy and Competitiveness grants TEC2012-34575 and UNPC10-4E-442, and by the Catalan Agency for
Support to Universities and Research (AGAUR) grant 2014 SGR 583.

\section{REFERENCES}

[1] Müller, D. et al., "Retrieval of Physical Particle Properties from Lidar Observations of Extinction and Backscatter at Multiple Wavelengths," Appl. Opt., vol. 37, no. 12, p. 2260, Apr. 1998.

[2] McNeil, W. R. and A. I. Carswell, "Lidar polarization studies of the troposphere.," Appl. Opt., vol. 14, no. 9, pp. 2158-68, Sep. 1975.

[3] Sassen, K., "Polarization in lidar," in Lidar. Range-Resolved Optical Remote Sensing of the Atmosphere, C. Weitkamp, Ed. Springer, 2005.

[4] Freudenthaler, V. et al., "Depolarization ratio profiling at several wavelengths in pure Saharan dust during SAMUM 2006," Tellus B, vol. 61, no. 1, Feb. 2009.

[5]De Tomasi F., and M. R. Perrone, "Multiwavelengths lidar to detect atmospheric aerosol properties," IET Sci. Meas. Technol., vol. 8, no. 3, pp. 143-149, May 2014.

[6]Biele, J., G. Beyerle, and G. Baumgarten, "Polarization Lidar: Correction of instrumental effects," Opt.Express, vol. 7, no. 12, pp. 427-435, 2000.

[7]Mattis, I., et al., "Systematic error of lidar profiles caused by a polarization-dependent receiver transmission: quantification and error correction scheme," Appl. Opt., vol. 48, no. 14, pp. 2742-2751, Oct. 2009.

[8] Mishchenko, M. I. and J. W. Hovenier, "Depolarization of light backscattered by randomly oriented nonspherical particles," Opt.Lett., vol. 20, no. 12, pp. 1356-1358, Jun. 1995.

[9]Flynn, C. J. et al. "Novel polarization-sensitive micropulse lidar measurement technique," Opt.Express, vol. 15, no. 6, pp. 2785-2790, Mar. 2007.

[10] Gimmestad, G. G., "Reexamination of depolarization in lidar measurements," Appl. Opt., vol. 47, no. 21, p. 3795, Jul. 2008.

[11] M. Born and E. Wolf, "Interference and diffraction with partially coherent light," in Principles of Optics, 6th ed., M. Born and E. Wolf, Eds. Pergamon Press, 1980, pp. 491-555. 